\title{
Transient electroosmotic flow of general Maxwell fluids through a slit microchannel
}

\author{
Yongjun Jian, Jie Su, Long Chang, Quansheng Liu and Guowei He
}

\begin{abstract}
Using Laplace transform method, semi-analytical solutions are presented for transient electroosmotic flow of Maxwell fluids between micro-parallel plates. The solution involves solving the linearized Poisson-Boltzmann equation, together with the Cauchy momentum equation and the Maxwell constitutive equation considering the depletion effect produced by the interaction between macro-molecules of the Maxwell fluids and the channel surface. The overall flow is divided into depletion layer and bulk flow outside of depletion layer. In addition, the Maxwell stress is incorporated to describe the boundary condition at the interface. The velocity expressions of these two layers were obtained respectively. By numerical computations of inverse Laplace transform, the influences of viscosity ratio $\mu$, density ratio $\rho$, dielectric constant ratio $\varepsilon$ of layer II to layer I, relaxation time $\bar{\lambda}_{1}$, interface charge density jump $Q$, and interface zeta potential difference $\Delta \bar{\psi}$ on transient velocity amplitude are presented.
\end{abstract}

Mathematics Subject Classification (2000). 76A05 - 76D05 - 76W05.

Keywords. Electric double layer (EDL) - Unsteady electroosmotic flow (EOF) · Maxwell fluids · Micro-parallel plates . Laplace transform.

\section{Introduction}

Microfluidics is one of the most important research areas in microelectromechanical systems (MEMS) due to its potential applications as a tool for studying fundamental physical and biochemical processes and a platform for performing chemical and biological assays [1,2]. Most solid surfaces acquire electrostatic charges when they are in contact with aqueous solution. The electrostatic charges on the solid surface will attract the counterions in the aqueous solution. Accumulation of these counterions forms an electrically charged layer, called electric double layer, near the solid-liquid interface [3]. The ions of opposite charge cluster immediately near the wall, forming the Stern layer, a layer of typical thickness of one ionic diameter. Right next to the Stern layer, the diffuse layer is formed, which contains both co-ions and counterions, and its ion density variation obeys the Boltzmann distribution [4]. The Stern layer and the diffuse layer constitute the EDL. When an external electric field is applied along the interface, the excess counterions in the EDL will be pushed to move and consequently causing the bulk liquid (the aqueous solution) to move due to the viscous effect. Such a liquid motion is referred to as the electroosmotic flow. EOF is a principal means to transport aqueous solutions in micron and submicron channels. Plug-like velocity profile is a signature of the pure EOF in a microchannel. The EOF can be easily controlled by the applied electric fields; hence, it has been widely used for precise liquid operation in intricate microchannel networks.

In the literature, various theoretical and experimental studies on steady EOF of Newtonian fluids in microchannels have been conducted under different geometric domains and physical conditions [5-10]. Recently, time-dependent EOF has been attracting growing attention as an alternative mechanism of microfluidic transport [11-17]. 
All the papers indicated above deal with Newtonian fluids. However, the flow behavior of non-Newtonian fluid is of high interest in many areas of science and technology. In practical applications for electroosmosis, such as sample collection, detection, mixing, and separation of various biological and chemical species on a chip integrated with fluidic pumps and valves, the fluid rheological behavior usually needs to be taken into account. The theoretical study of electroosmotic flows of non-Newtonian fluids is recent and has been mostly limited to simple fluid models due to the complex constitutive relations.

Limited studies have been reported for EOF involving non-Newtonian fluids. The EOF of power-law fluids was studied in Refs [18-22]. It was found that the behavior index $n$ of the power-law fluid substantially influences velocity profiles. EOF of non-Newtonian fluids with viscoelasticity was also addressed analytically [23-28]. It was revealed that the presence of the viscoelasticity can essentially affect the flow pattern and the volumetric flow rate.

It needs to note that all the above models implicitly assume that fluid properties are uniform in the whole domain for viscoelastic fluids. However, such a situation is hardly found in experiments. In fact, due to the unavoidable interaction between macro-molecules and the channel surface, polymer depletion and adsorption phenomena will be produced [29]. Sousa et al. [30] investigated the effects of the depletion layer on electroosmotic Poiseuille flows of PTT (Phan-Thien-Tanner) fluids. Liu et al. [31] studied time periodic EOF of Jeffrey fluid in a slit microchannel taking the depletion effect into account. However, these theoretical analyses overlooked the effect of electric field generated stress (Maxwell stress) at the interface between the depletion layer and bulk flow outside of depletion layer. Volkov et al. [32] pointed out that at the interface of two immiscible electrolyte solutions, a narrow region exists where the electric potential changes abruptly due to the adsorption of ions. The sharp change at this layer can often be described by a zeta potential jump across the interface. Theoretical studies on steady two-fluid EOF with full interfacial electrostatics [33] show the importance of the Maxwell stress on interface. Very recently, the free surface instability in EOF of ultrathin liquid films was investigated by Mayur et al. [34]. The role of interfacial Maxwell stress on thin film stability is highlighted.

Although some basic features of EOF of non-Newtonian fluid are reported in the aforementioned studies, much of its rich nature remains to be examined. Moreover, few publications investigate the transient EOF in non-Newtonian fluid. The main purpose of this paper is extending our early work [31] to study the transient EOF of Maxwell fluids in a slit microchannel with the depletion effects included. The overall flow is divided into depletion layer (taking as Newtonian fluid) and bulk flow outside of depletion layer (taking as Maxwell fluid). We incorporate the Maxwell stress on the interface. Semi-analytical solutions for EOF velocity are obtained for each layer by solving the general Cauchy momentum equation with Laplace transform. Furthermore, the influencing parameters for EOF of Maxwell fluids are analyzed.

This paper is organized as follows: The physical description of the problem and the analytical solution to the equations governing the transient EOF of the Maxwell model are presented in the Sect. 2. The Sect. 3 discusses the numerical results of the study and the parametric dependence of the EOF velocity. Finally, conclusions are presented in the Sect. 4.

\section{Problem formulation}

\subsection{Electrical potential equation}

The transient EOF of the incompressible Maxwell fluids between two negatively charged micro-parallel plates separated by a distance (height) $2 H$ is sketched in Fig. 1 . The length of the channel is $L$ and the width is $W$, both assumed to be much larger than the height, i.e., $L, W>>2 H$. Use a two-dimensional coordinate system where $y$-axis and $x$-axis are perpendicular and tangential to the charged surface, respectively. The bottom plate is located at $y=-H$, while the top plate is located at $y=H$. Due to symmetry of the geometry and flow conditions with respect to the channel mid-plane $(y=0)$, only 


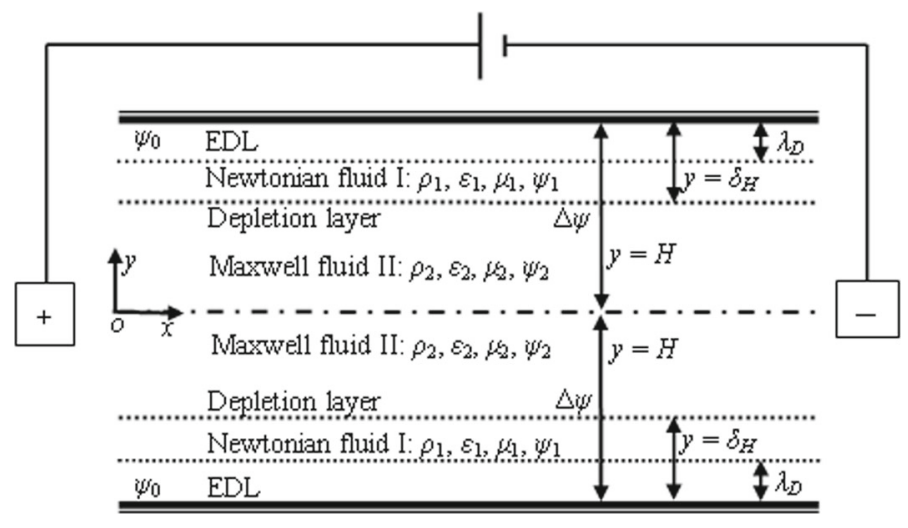

FIG. 1. Schematic of transient EOF of the Maxwell fluids through a slit microchannel

the upper half of the channel $(0 \leq y \leq H)$ is considered in this analysis. The chemical interaction of electrolyte liquid and solid wall generates an EDL, a very thin charged liquid layer the thickness $\xi$ at the solid-liquid interface. Due to the interaction between macro-molecules and the channel surfaces, the concentration of nonadsorbing particles decreases steeply near smooth surfaces, leaving a depleted layer of pure solvent adjacent to the solid-liquid interface. The depletion layer has a thickness $\delta_{H}$, which is larger than the EDL thickness and much smaller than half height $H$ of the microchannel (see Fig. 1). Within the depletion layer (noted as layer I), the viscosity of the solvent is much lower than that of the bulk and the fluid can be taken as Newtonian fluid, while the bulk fluids out of the depletion layer are modeled by Maxwell viscoelastic model (noted as layer II).

The EOF is pumped by an axial DC electric field with strength $E_{0}$, the liquid inside the EDL sets in motion along $x$ direction due to electroosmosis. For a symmetric binary electrolyte solution, the electrical potential and the local volumetric net charge density within layer I and layer II are described by the following Poisson-Boltzmann (PB) equations ( $i=1$ means layer I, $i=2$ means layer II)

$$
\begin{aligned}
\frac{\mathrm{d}^{2} \psi_{i}(y)}{\mathrm{d} y^{2}} & =-\frac{\rho_{e i}(y)}{\varepsilon_{i}}, \quad i=1,2 \\
\rho_{e_{i}}(y) & =-2 n_{0} z_{\nu} e_{0} \sinh \left[\frac{z_{\nu} e_{0} \psi_{i}(y)}{k_{b} T}\right], \quad i=1,2
\end{aligned}
$$

where $\psi_{i}(y), \rho_{e i}(y)$, and $\varepsilon_{i}$ are the electrical potential, the local volumetric net charge density and dielectric constant of the electrolyte liquid for layer I and layer II, respectively, $n_{0}$ is the ion density of bulk liquid, $z_{\nu}$ is the valence, $e_{0}$ is the electron charge, $k_{b}$ is the Boltzmann constant, and $T$ is the absolute temperature.

Assuming the electrical potential is small enough, linear approximation can be used for the hyperbolic sine function appearing in the right-hand side of Eq. (2.2), by inserting Eq. (2.2) into Eq. (2.1), resulting in the so-called Debye-Hückel equation

$$
\frac{\mathrm{d}^{2} \psi_{i}(y)}{\mathrm{d} y^{2}}=\frac{2 n_{0} z_{\nu}^{2} e_{0}^{2}}{\varepsilon_{i} k_{b} T} \psi_{i}(y)=\kappa_{i}^{2} \psi_{i}(y) \quad \text { with } \quad \kappa_{i}=\left(2 n_{0} z_{\nu}^{2} e_{0}^{2} / \varepsilon_{i} k_{b} T\right)^{1 / 2}, \quad i=1,2
$$

where $\lambda_{D i}=1 / \kappa_{i}$ has the dimension of length and denotes the EDL thickness. The boundary conditions for the potential are usually written in the form

$$
\begin{aligned}
\left.\psi_{1}(y)\right|_{y=H} & =\psi_{0}, \\
\left.\frac{\mathrm{d} \psi_{2}(y)}{\mathrm{d} y}\right|_{y=0} & =0,
\end{aligned}
$$


here, $\psi_{0}$ is the wall zeta potential. Moreover, at the interface $\left(y=H-\delta_{H}\right)$ between layer I and layer II, two boundary conditions are required. Here, we impose the zeta potential difference $\Delta \psi$ and Gauss's law for the electrical displacement

$$
\begin{array}{r}
{\left.\left[\psi_{2}(y)-\psi_{1}(y)\right]\right|_{y=H-\delta_{H}}=\Delta \psi,} \\
{\left.\left[\varepsilon_{1} \frac{\mathrm{d} \psi_{1}(y)}{\mathrm{d} y}-\varepsilon_{2} \frac{\mathrm{d} \psi_{2}(y)}{\mathrm{d} y}\right]\right|_{y=H-\delta_{H}}=-q_{s},}
\end{array}
$$

Here, $q_{s}$ denotes interface charge density jump. Introducing the following dimensionless groups:

$$
\bar{y}=\frac{y}{H}, D e_{i}=\frac{\lambda_{D i}}{H},\left[\bar{\psi}_{i}(\bar{y}), \bar{\psi}_{0}, \Delta \bar{\psi}\right]=\frac{z_{\nu} e_{0}}{k_{b} T}\left[\psi_{i}(y), \psi_{0}, \Delta \psi\right], \quad Q=\frac{H z_{\nu} e_{0} q_{s}}{\varepsilon_{1} k_{b} T},
$$

where $D e_{i}$ is the nondimensional Debye length. Dimensionless electrical potential Eq. (2.3) and the corresponding boundary conditions (2.4) can be written as

$$
\begin{gathered}
\frac{\mathrm{d}^{2} \bar{\psi}_{i}(\bar{y})}{\mathrm{d} y^{2}}=\frac{1}{D e_{i}^{2}} \bar{\psi}_{i}(\bar{y}), \\
\left.\bar{\psi}_{1}(\bar{y})\right|_{\bar{y}=1}=\bar{\psi}_{0},\left.\frac{\mathrm{d} \bar{\psi}_{2}(\bar{y})}{\mathrm{d} \bar{y}}\right|_{\bar{y}=0}=0,\left.\quad\left[\bar{\psi}_{2}(\bar{y})-\bar{\psi}_{1}(\bar{y})\right]\right|_{\bar{y}=1-\bar{\delta}_{H}}=\Delta \bar{\psi} \\
\left.\left(\frac{\mathrm{d} \bar{\psi}_{1}(\bar{y})}{\mathrm{d} \bar{y}}-\varepsilon \frac{\mathrm{d} \bar{\psi}_{2}(\bar{y})}{\mathrm{d} \bar{y}}\right)\right|_{\bar{y}=1-\bar{\delta}_{H}}=-Q \quad \text { with } \quad \varepsilon=\frac{\varepsilon_{2}}{\varepsilon_{1}} \quad \text { and } \quad \bar{\delta}_{H}=\frac{\delta_{H}}{H} .
\end{gathered}
$$

The general solutions of Eq. (2.6) are obtained as

$$
\bar{\psi}_{1}(\bar{y})=A_{1} \cosh \left(\frac{1}{D e_{1}} \bar{y}\right)+B_{1} \sinh \left(\frac{1}{D e_{1}} \bar{y}\right), \quad \bar{\psi}_{2}(\bar{y})=A_{2} \cosh \left(\frac{1}{D e_{2}} \bar{y}\right)+B_{2} \sinh \left(\frac{1}{D e_{2}} \bar{y}\right) .
$$

With the boundary condition (2.7), the constants $A_{1}, B_{1}, A_{2}$, and $B_{2}$ can be written as

$$
\begin{aligned}
B_{2}= & 0 \\
B_{1}= & \left\{\bar{\psi}_{0} \csc h \frac{1}{D e_{1}}\left[\frac{1}{D e_{1}} \operatorname{coth} \frac{1-\bar{\delta}_{H}}{D e_{2}}-\frac{\varepsilon}{D e_{2}} \operatorname{coth} \frac{1-\bar{\delta}_{H}}{D e_{1}}\right]-\operatorname{coth} \frac{1}{D e_{1}}\left[\frac{\varepsilon \Delta \bar{\psi}}{D e_{2}}\right.\right. \\
& \left.\left.\times \csc h \frac{1-\bar{\delta}_{H}}{D e_{1}}-Q \operatorname{coth} \frac{1-\bar{\delta}_{H}}{D e_{2}} \operatorname{csch} \frac{1-\bar{\delta}_{H}}{D e_{1}}\right]\right\} /\left\{\frac{1}{D e_{1}} \operatorname{coth} \frac{1-\bar{\delta}_{H}}{D e_{2}}\right. \\
& \left.-\varepsilon \frac{1}{D e_{2}} \operatorname{coth} \frac{1-\bar{\delta}_{H}}{D e_{1}}-\operatorname{coth} \frac{1}{D e_{1}}\left[\frac{1}{D e_{1}} \operatorname{coth} \frac{1-\bar{\delta}_{H}}{D e_{1}} \operatorname{coth} \frac{1-\bar{\delta}_{H}}{D e_{2}}-\frac{\varepsilon}{D e_{2}}\right]\right\}, \\
A_{1}= & \bar{\psi}_{0} \operatorname{sech} \frac{1}{D e_{1}}-B_{1} \tanh \frac{1}{D e_{1}}, \\
A_{2}= & \operatorname{sech} \frac{1-\bar{\delta}_{H}}{D e_{2}}\left\{\Delta \bar{\psi}+A_{1} \cosh \frac{1-\bar{\delta}_{H}}{D e_{1}}+B_{1} \sinh \frac{1-\bar{\delta}_{H}}{D e_{1}}\right\} .
\end{aligned}
$$

\subsection{Velocity distributions}

If we consider only pure EOF along $x$ direction, the one-dimensional Cauchy momentum equation can be expressed as

$$
\rho_{i} \frac{\partial u^{i}(y, t)}{\partial t}=-\frac{\partial}{\partial y}\left(\tau_{y x}^{i}\right)+\rho_{e i}(y) E_{0},
$$


where $u(y, t)$ is the axial velocity along positive $x$ direction, $\rho_{i}$ is the fluid density, $t$ is time, $\tau_{y x}$ is the stress tensor, and $E_{0}$ is DC electric field. Within the layer I, the stress tensor can be described by Newtonian fluid with viscosity $\mu_{1}$

$$
\tau_{y x}^{I}=-\mu_{1} \frac{\partial u^{I}(y, t)}{\partial y}
$$

where $u^{I}(y, t)$ and $\tau_{y x}^{I}$ mean the velocity and stress tensor in layer I, respectively, whereas the viscoelastic fluid within the layer II is described by Maxwell model, and its constitutive equation satisfies

$$
\tau_{y x}^{I I}+\lambda_{1} \frac{\partial \tau_{y x}^{I I}}{\partial t}=-\mu_{2} \frac{\partial u^{I I}(y, t)}{\partial y}
$$

where $\lambda_{1}$ is the relaxation time, $\mu_{2}$ is the zero shear rate viscosity, $u^{I I}(y, t)$ and $\tau_{y x}^{I I}$ mean the velocity and stress tensor in layer II, respectively. For layer I, no-slip condition at the wall should be satisfied

$$
\left.u^{I}(y, t)\right|_{y=H}=0 .
$$

At the interface between the depletion layer and the bulk of the fluid, both fluids move at the same velocity

$$
\left.u^{I}(y, t)\right|_{y=H-\delta_{H}}=\left.u^{I I}(y, t)\right|_{y=H-\delta_{H}} .
$$

For layer II, besides velocity continue condition of Eq. (2.11), the centerplane flow symmetry condition should be satisfied due to only half of the channel being considered

$$
\left.\frac{\partial u^{I I}(y, t)}{\partial y}\right|_{y=0}=0 \text {. }
$$

In addition, the total stresses including shear stress and Maxwell stress at the interface between layer I and layer II should be equal

$$
\tau_{y x}^{I}+\varepsilon_{1} E_{0} \frac{\mathrm{d} \psi_{1}(y)}{\mathrm{d} y}=\tau_{y x}^{I I}+\varepsilon_{2} E_{0} \frac{\mathrm{d} \psi_{2}(y)}{\mathrm{d} y} .
$$

The above problem is reformulated in terms of the following nondimensional variables defined by

$$
\begin{aligned}
\left(\bar{y}, \bar{\delta}_{H}\right) & =\frac{\left(y, \delta_{H}\right)}{H}, \quad\left(\bar{t}, \bar{\lambda}_{1}\right)=\frac{\left(t, \lambda_{1}\right)}{\rho_{1} H^{2} / \mu_{1}}, \quad\left[\bar{u}^{I}(\bar{y}, \bar{t}), \bar{u}^{I I}(\bar{y}, \bar{t})\right]=\frac{\left[u^{I}(y, t), u^{I I}(y, t)\right]}{U_{e o}}, \\
U_{e o} & =-\frac{\varepsilon_{1} \psi_{0} E_{0}}{\mu_{1}}, \quad\left(\bar{\tau}_{\bar{y} \bar{x}}^{I}, \bar{\tau}_{\bar{y} \bar{x}}^{I I}\right)=\frac{\left(\tau_{y x}^{I}, \tau_{y x}^{I I}\right)}{\mu_{1} U_{e o} / H}, \quad \mu=\frac{\mu_{2}}{\mu_{1}}, \quad \rho=\frac{\rho_{2}}{\rho_{1}},
\end{aligned}
$$

where $U_{e o}$ denotes steady Helmholtz-Smoluchowski EOF velocity of Newtonian fluids within the layer I, $\mu$ and $\rho$ are viscosity and density ratios of layer II to layer I.

For layer I, using Eq. (2.11), the electroosmosis Eq. (2.10) and corresponding boundary conditions (2.13)-(2.14) are normalized as

$$
\begin{gathered}
\frac{\partial \bar{u}^{I}(\bar{y}, \bar{t})}{\partial \bar{t}}=\frac{\partial^{2} \bar{u}^{I}(\bar{y}, \bar{t})}{\partial \bar{y}^{2}}+\frac{1}{D e_{1}^{2}} \frac{\bar{\psi}_{1}}{\bar{\psi}_{0}}, \\
\left.\bar{u}^{I}(\bar{y}, \bar{t})\right|_{\bar{y}=1}=0,\left.\bar{u}^{I}(\bar{y}, \bar{t})\right|_{\bar{y}=1-\bar{\delta}_{H}}=\left.\bar{u}^{I I}(\bar{y}, \bar{t})\right|_{\bar{y}=1-\bar{\delta}_{H}} .
\end{gathered}
$$

For layer II, the normalized electroosmosis Eq. (2.10), constitutive Eq. (2.12), and corresponding boundary conditions $(2.15)-(2.16)$ are 


$$
\begin{gathered}
\rho \frac{\partial \bar{u}^{I I}(\bar{y}, \bar{t})}{\partial \bar{t}}=-\frac{\partial \bar{\tau}_{\bar{y} \bar{x}}^{I I}}{\partial \bar{y}}+\frac{\varepsilon}{D e_{2}^{2}} \frac{\bar{\psi}_{2}}{\bar{\psi}_{0}}, \\
\bar{\tau}_{\bar{y} \bar{x}}^{I I}+\bar{\lambda}_{1} \frac{\partial \bar{\tau}_{\bar{y} \bar{x}}^{I I}}{\partial \bar{t}}=-\mu \frac{\partial \bar{u}^{I I}}{\partial \bar{y}}, \\
\left.\frac{\partial \bar{u}^{I I}(\bar{y}, \bar{t})}{\partial \bar{y}}\right|_{\bar{y}=0}=0,\left.\quad\left[\bar{\tau}_{\bar{y} \bar{x}}^{I}-\frac{1}{\bar{\psi}_{0}} \frac{d \bar{\psi}_{1}}{d \bar{y}}\right]\right|_{\bar{y}=1-\bar{\delta}_{H}}=\left.\left[\bar{\tau}_{\bar{y} \bar{x}}^{I I}-\frac{\varepsilon}{\bar{\psi}_{0}} \frac{d \bar{\psi}_{2}}{d \bar{y}}\right]\right|_{\bar{y}=1-\bar{\delta}_{H} .}
\end{gathered}
$$

Let us employ the method of Laplace transform defined by

$$
\begin{aligned}
& U^{I}(\bar{y}, s)=L\left[\bar{u}^{I}(\bar{y}, \bar{t})\right]=\int_{0}^{\infty} \bar{u}^{I}(\bar{y}, \bar{t}) e^{-s \bar{t}} \mathrm{~d} \bar{t}, \\
& U^{I I}(\bar{y}, s)=L\left[\bar{u}^{I I}(\bar{y}, \bar{t})\right]=\int_{0}^{\infty} \bar{u}^{I I}(\bar{y}, \bar{t}) e^{-s \bar{t}} \mathrm{~d} \bar{t} \\
& \overline{\bar{\tau}}_{\bar{y} \bar{x}}^{I I}(\bar{y}, s)=L\left[\bar{\tau}_{\bar{y} \bar{x}}^{I I}(\bar{y}, \bar{t})\right]=\int_{0}^{\infty} \bar{\tau}_{\bar{y} \bar{x}}^{I I}(\bar{y}, \bar{t}) e^{-s \bar{t}} \mathrm{~d} \bar{t} .
\end{aligned}
$$

Assuming initial condition satisfies

$$
u^{I}(y, 0)=u^{I I}(y, 0)=u_{t}^{I}(y, 0)=u_{t}^{I I}(y, 0)=0,
$$

then for layer I, the Laplace transforms of Eq. (2.18) and boundary condition (2.19) give

$$
\begin{gathered}
\frac{\partial^{2} U^{I}(\bar{y}, s)}{\partial \bar{y}^{2}}=s U^{I}(\bar{y}, s)-\frac{1}{D e_{1}^{2}} \frac{\bar{\psi}_{1}}{s \bar{\psi}_{0}} \\
\left.U^{I}(\bar{y}, s)\right|_{\bar{y}=1}=0,\left.\quad U^{I}(\bar{y}, s)\right|_{\bar{y}=1-\bar{\delta}_{H}}=\left.U^{I I}(\bar{y}, s)\right|_{\bar{y}=1-\bar{\delta}_{H}} .
\end{gathered}
$$

For layer II, the transforms of Eqs. (2.20) and (2.21) and boundary condition (2.22) give

$$
\begin{gathered}
\rho s U^{I I}(\bar{y}, s)=-\frac{\partial \overline{\bar{\tau}}_{\bar{y} \bar{x}}}{\partial \bar{y}}+\frac{\varepsilon}{D e_{2}^{2}} \frac{\bar{\psi}_{2}}{s \bar{\psi}_{0}}, \\
\left(1+\bar{\lambda}_{1} s\right) \overline{\bar{\tau}}_{\bar{y} \bar{x}}^{I I}=-\mu \frac{\partial U^{I I}(\bar{y}, s)}{\partial \bar{y}}, \\
\left.\frac{\partial U^{I I}(\bar{y}, s)}{\partial \bar{y}}\right|_{\bar{y}=0}=0, \\
{\left.\left[\frac{\partial U^{I}(\bar{y}, s)}{\partial \bar{y}}+\frac{1}{\bar{\psi}_{0} s} \frac{\mathrm{d} \bar{\psi}_{1}}{\mathrm{~d} \bar{y}}\right]\right|_{\bar{y}=1-\bar{\delta}_{H}}=\left.\left[\frac{\mu}{\left(1+\lambda_{1} s\right)} \frac{\partial U^{I I}(\bar{y}, s)}{\partial \bar{y}}+\varepsilon \frac{1}{\bar{\psi}_{0} s} \frac{\mathrm{d} \bar{\psi}_{2}}{\mathrm{~d} \bar{y}}\right]\right|_{\bar{y}=1-\bar{\delta}_{H}} .}
\end{gathered}
$$

Eliminating $\overline{\bar{\tau}} \overline{\bar{y}} \bar{I}$ by combining Eqs. (2.27) and (2.28) yields

$$
\frac{\partial^{2} U^{I I}(\bar{y}, s)}{\partial \bar{y}^{2}}=\beta^{2} U^{I I}(\bar{y}, s)-\frac{\varepsilon\left(1+\bar{\lambda}_{1} s\right)}{D e_{2}^{2} \mu} \frac{\bar{\psi}_{2}}{s \bar{\psi}_{0}} \quad \text { with } \quad \beta^{2}=\frac{\rho}{\mu} s\left(1+\bar{\lambda}_{1} s\right) .
$$

Equations (2.25) and (2.31) for layer I and layer II are linear and inhomogeneous ordinary differential equation, and their solutions are

$$
\begin{aligned}
& U^{I}(\bar{y}, s)=C_{1} \cosh (\sqrt{s} \bar{y})+C_{2} \sinh (\sqrt{s} \bar{y})+C \cosh \left(\frac{1}{D e_{1}} \bar{y}\right)+D \sinh \left(\frac{1}{D e_{1}} \bar{y}\right), \\
& U^{I I}(\bar{y}, s)=C_{3} \cosh (\beta \bar{y})+C_{4} \sinh (\beta \bar{y})+E \cosh \left(\frac{1}{D e_{2}} \bar{y}\right) .
\end{aligned}
$$


Using boundary conditions (2.26) and (2.29), these constants can be determined as

$$
\begin{aligned}
& C=\frac{A_{1}}{s \bar{\psi}_{0} D e_{1}^{2}\left(s-1 / D e_{1}^{2}\right)}, \quad D=\frac{B_{1}}{s \bar{\psi}_{0} D e_{1}^{2}\left(s-1 / D e_{1}^{2}\right)}, \quad E=\frac{\varepsilon\left(1+\bar{\lambda}_{1} s\right) A_{2}}{\mu s \bar{\psi}_{0} D e_{2}^{2}\left(\beta^{2}-1 / D e_{2}^{2}\right)}, \\
& C_{1}=\left\{\operatorname { s i n h } ( \sqrt { s } ) \operatorname { c o s h } ( e ) \left[\frac{\mu}{\left(1+\bar{\lambda}_{1} s\right) D e_{2}} E \sinh \left(a_{2}\right)-C \frac{1}{D e_{1}} \sinh \left(a_{1}\right)-D \frac{1}{D e_{1}} \cosh \left(a_{1}\right)\right.\right. \\
&\left.+\frac{1}{s \bar{\psi}_{0}}\left[\frac{\varepsilon A_{2}}{D e_{2}} \sinh \left(a_{2}\right)-\frac{A_{1}}{D e_{1}} \sinh \left(a_{1}\right)-\frac{B_{1}}{D e_{1}} \cosh \left(a_{1}\right)\right]\right]-\frac{\mu \beta}{1+\bar{\lambda}_{1} s} \sinh (e) \sinh (\sqrt{s}) \\
& \times\left[E \cosh \left(a_{2}\right)-C \cosh \left(a_{1}\right)-D \sinh \left(a_{1}\right)\right] \\
&\left.+\left[\sqrt{s} \cosh (e) \cosh (b)-\frac{\mu \beta}{1+\bar{\lambda}_{1} s} \sinh (e) \sinh (b)\right] \cdot\left[C \cosh \frac{1}{D e_{1}}+D \sinh \frac{1}{D e_{1}}\right]\right\} / \\
&\left\{\sinh (\sqrt{s})\left[\sqrt{s} \cosh (e) \sinh (b)-\frac{\mu \beta}{1+\bar{\lambda}_{1} s} \sinh (e) \cosh (b)\right]\right. \\
&\left.-\cosh (\sqrt{s})\left[\sqrt{s} \cosh (e) \cosh (b)-\frac{\mu \beta}{1+\bar{\lambda}_{1} s} \sinh (e) \sinh (b)\right]\right\} \\
& C_{2}=-\operatorname{csch}(\sqrt{s})\left[C \cosh \frac{1}{D e_{1}}+D \sinh \frac{1}{D e_{1}}+C_{1} \cosh (\sqrt{s})\right], \\
& C_{3}=\sec h(e)\left[C_{1} \cosh (b)+C_{2} \sinh (b)+C \cosh \left(a_{1}\right)+D \sinh \left(a_{1}\right)-E \cosh \left(a_{2}\right)\right], \\
& C_{4}=0,
\end{aligned}
$$

where

$$
a_{1}=\left(1-\bar{\delta}_{H}\right) / D e_{1}, \quad a_{2}=\left(1-\bar{\delta}_{H}\right) / D e_{2}, \quad b=\sqrt{s}\left(1-\bar{\delta}_{H}\right), \quad e=\beta\left(1-\bar{\delta}_{H}\right)
$$

The inverse Laplace transform is defined by

$$
\begin{gathered}
\bar{u}^{I}(\bar{y}, \bar{t})=L^{-1}\left[U^{I}(\bar{y}, s)\right]=\frac{1}{2 \pi i} \int_{\Gamma_{1}} U^{I}(\bar{y}, s) e^{s \bar{t}} \mathrm{~d} s, \\
\bar{u}^{I I}(\bar{y}, \bar{t})=L^{-1}\left[U^{I I}(\bar{y}, s)\right]=\frac{1}{2 \pi i} \int_{\Gamma_{2}} U^{I I}(\bar{y}, s) e^{s \bar{t}} \mathrm{~d} s,
\end{gathered}
$$

where $\Gamma_{1}$ and $\Gamma_{2}$ are vertical line to the right of all singularities of $U^{I}$ and $U^{I I}$ in the complex $s$ plane. Due to the complexity of the express of $U^{I}$ and $U^{I I}$, the numerical computation must be performed by numerical inverse Laplace transform.

\section{Numerical results and discussion}

In the previous section, we have obtained semi-analytical transient EOF velocity of generalized Maxwell fluids which depends mainly on some nondimension parameters, such as viscosity ratio $\mu$, density ratio $\rho$, dielectric constant ratio $\varepsilon$ of layer II to layer I, relaxation time $\bar{\lambda}_{1}$, interface charge density jump $Q$, and interface zeta potential difference $\Delta \bar{\psi}$. In this section, we will discuss their influence on the transient EOF velocity in detail. In the following computations, the normalized thickness of the depletion layer $\bar{\delta}_{H}$ is taken as 0.1 [29] and $\bar{\psi}_{0}$ is taken as 1 . The numerical inversion of Laplace transforms is based on 


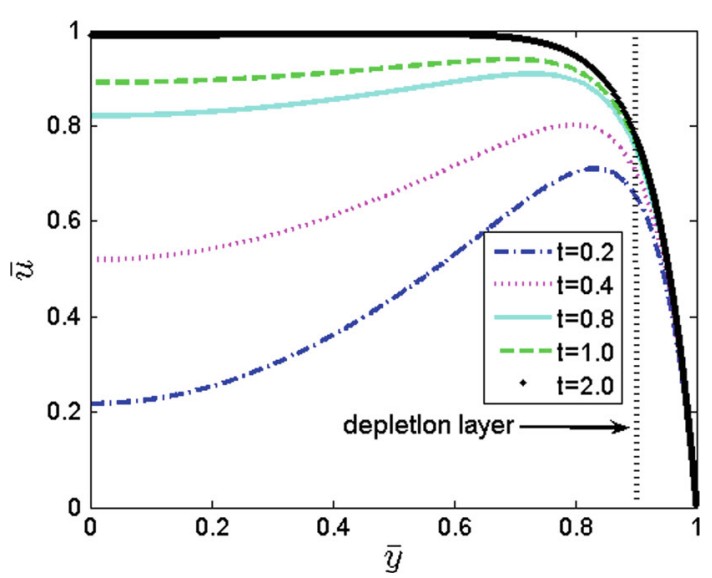

FIG. 2. Simplified transient velocities for Newtonian fluids $\left(1 / D e_{2}=15,1 / D e_{1}=\varepsilon^{1 / 2} / D e_{2}, \varepsilon=1, \bar{\delta}_{H}=0.1, \rho=1, \mu=1\right.$, $\left.\bar{\lambda}_{1}=0, \Delta \bar{\psi}=0, Q=0\right)$

(a)

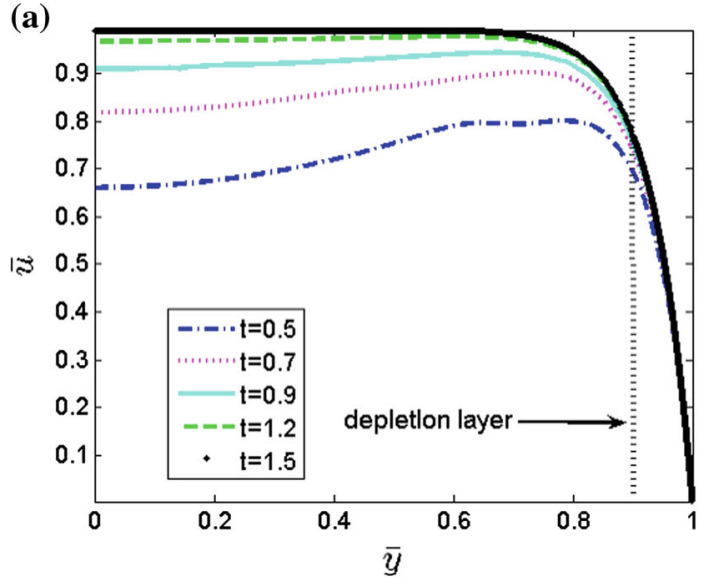

(c)

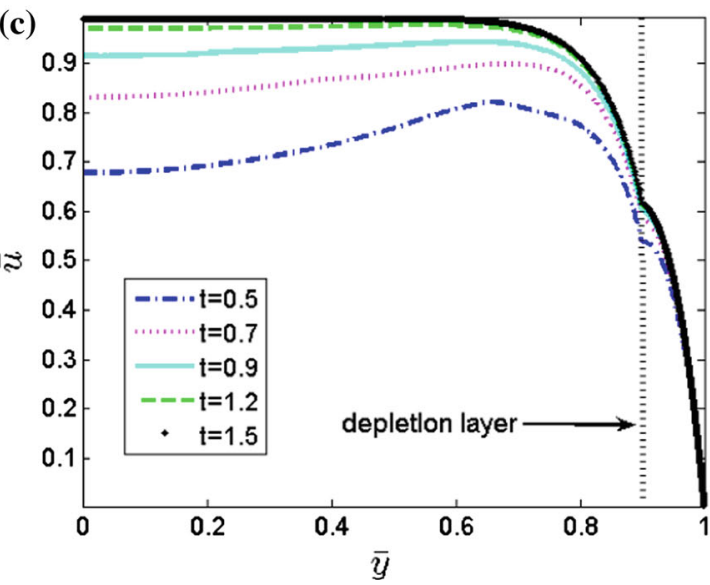

(b)

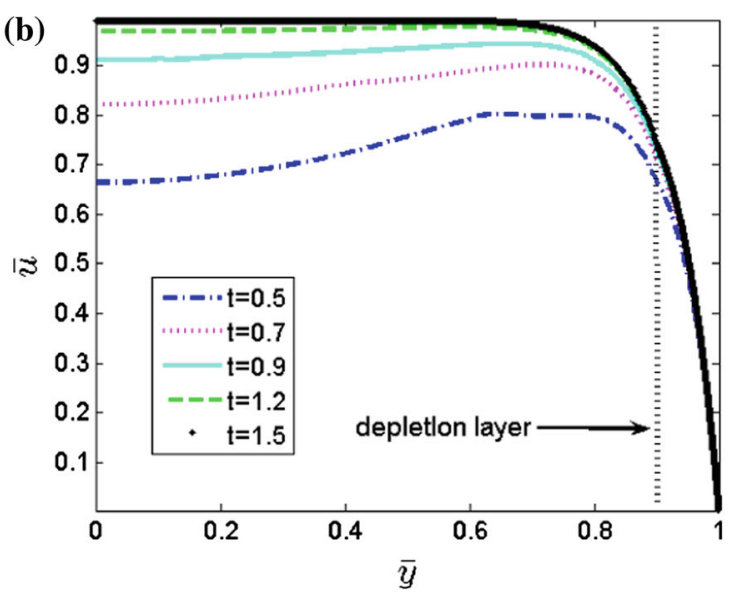

(d)

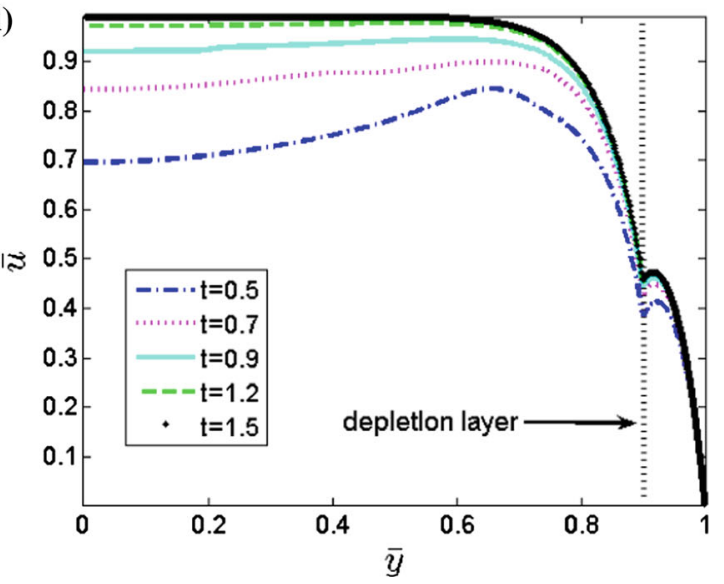

FIG. 3. Effects of the dimensionless interface charge density jump $Q$ on the normalized transient EOF velocity of Maxwell fluids $\left(1 / D e_{2}=15,1 / D e_{1}=\varepsilon^{1 / 2} / D e_{2}, \varepsilon=1, \rho=1, \mu=1, \bar{\lambda}_{1}=0.1, \Delta \bar{\psi}=0\right): \mathbf{a} Q=0.1, \mathbf{b} Q=1, \mathbf{c} Q=5, \mathbf{d} Q=10$ 
(a)
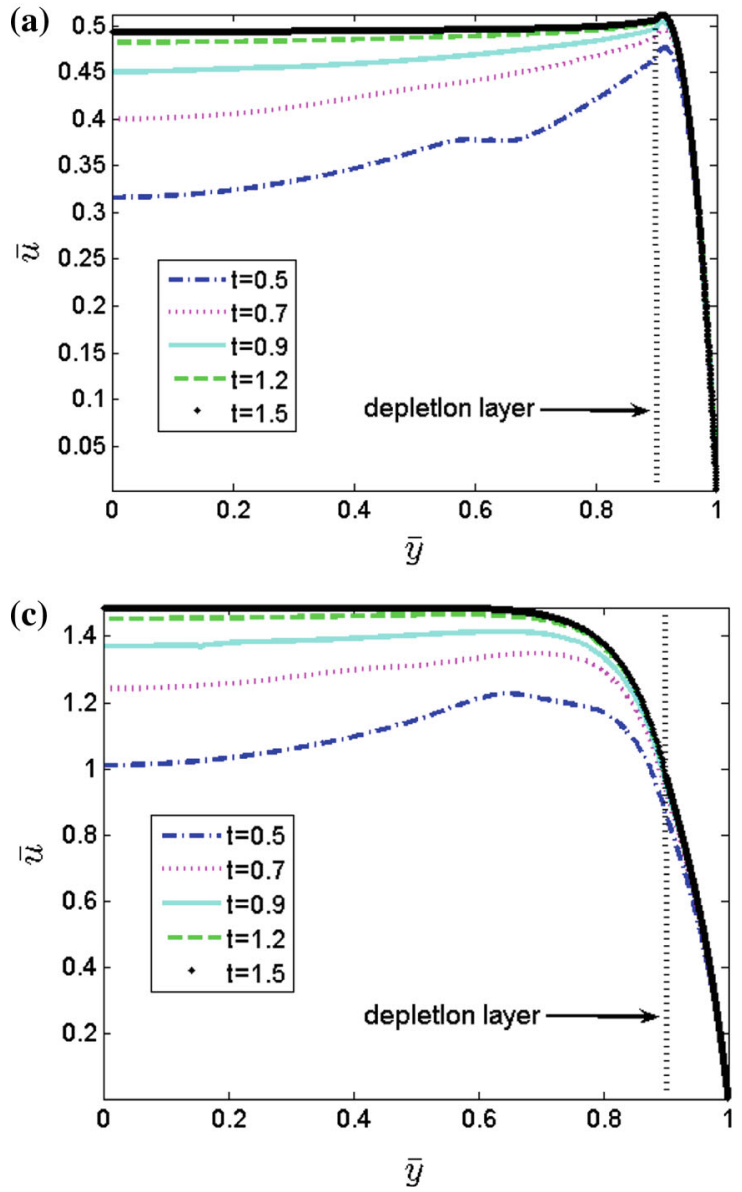

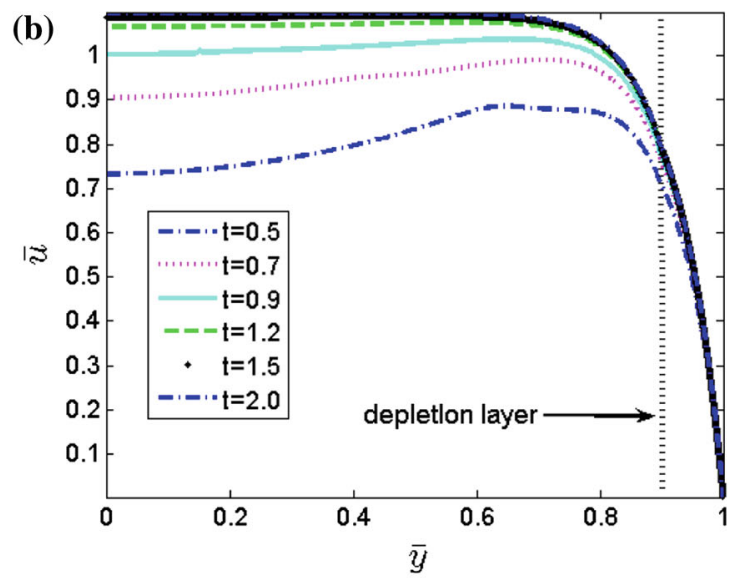

(d)

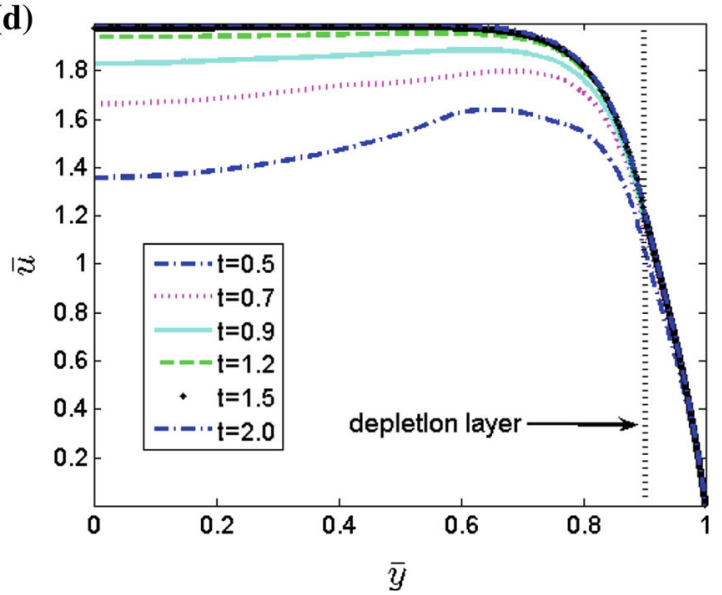

FIG. 4. Influences of the interface zeta potential difference $\Delta \bar{\psi}$ on the transient EOF velocity of Maxwell fluids $\left(1 / D e_{2}=\right.$ $\left.15,1 / D e_{1}=\varepsilon^{1 / 2} / D e_{2}, \mu=1, \rho=1, \bar{\lambda}_{1}=0.1, Q=1, \varepsilon=1\right): \mathbf{a} \Delta \bar{\psi}=-0.5, \mathbf{b} \Delta \bar{\psi}=0.1, \mathbf{c} \Delta \bar{\psi}=0.5, \mathbf{d} \Delta \bar{\psi}=1$

accelerating the convergence of the Fourier series obtained from the inversion integral using trapezoidal rule, which was proposed by De Hood et al. [35].

When $\rho=1, \mu=1, \varepsilon=1, \bar{\lambda}_{1}=0, \Delta \bar{\psi}=0$, and $Q=0$, current theoretical results for Maxwell fluids lead to the case of Newtonian fluids. Figure 2 shows the transient velocity for Newtonian fluids. It can be noted from Fig. 2 that as expected, a classical plug-like velocity profile can be obtained when the velocity distribution attains steady status.

The effects of the dimensionless interface charge density jump $Q(Q=0.1, Q=1, Q=5, Q=10)$ on the transient EOF velocity of Maxwell fluids are illustrated in Fig. 3 for given values of all the other parameters. With the increase in interface charge density jump $Q$, the velocity shows a strong jump at the interface of the depletion layer.

Figure 4 shows the influences of the interface zeta potential difference $\Delta \bar{\psi}(-0.5,0.1,0.5,1)$ on the transient EOF velocity of Maxwell fluids. At the interface of two immiscible electrolyte solutions, a narrow region exists where the electric potential changes abruptly due to the adsorption of ions. It can be found from Fig. 4 that as expected, the velocity amplitude becomes large with the interface zeta potential difference under steady status. 

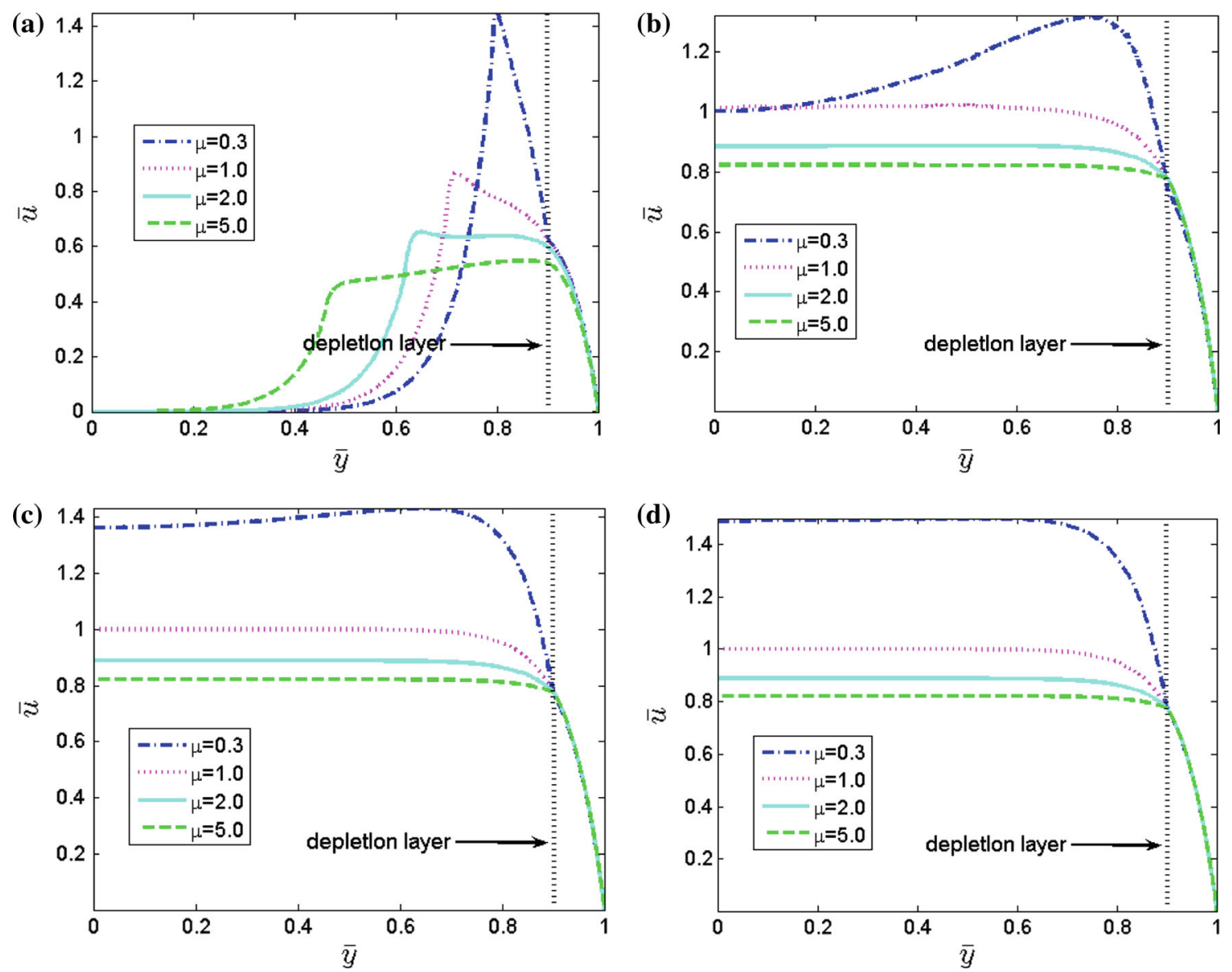

FIG. 5. Influences of the viscosity ratio $\mu$ on the transient EOF velocity of Maxwell fluids $\left(1 / D e_{2}=15,1 / D e_{1}=\right.$ $\left.\varepsilon^{1 / 2} / D e_{2}, \Delta \bar{\psi}=0, \rho=1, \bar{\lambda}_{1}=0.2, Q=0, \varepsilon=1\right): \mathbf{a} t=0.1, \mathbf{b} t=1.4, \mathbf{c} t=2.5, \mathbf{d} t=4.0$

Figure 5 shows the effects of viscosity ratio $\mu(0.3,1.0,2.0,5.0)$ of layer II to layer I on the EOF velocity of Maxwell fluids for different times. When the external electric field is applied at both ends of microchannel, the ions in EDL will be subjected to move because of the electric field force. Due to the effect of fluid viscosity, the freedom ions will drive the fluid particles nearby to move. With the increase in time, the fluid's movement will spread to the whole microchannel. For smaller viscosity ratio $\mu$, it means the viscosity of layer II is small, the fluid is easy to drive, so the fluid velocity is large. Similar explanation can be used for larger viscosity ratio. Velocity profiles of EOF are strongly dependent on the viscosity ratio $\mu$. When the viscosity ratio is high, the flow resistance of the low EO mobility liquid is high, resulting in the velocity amplitude becomes small. It can be shown from Fig. 5 that as expected, the velocity amplitude becomes small with the increase in the viscosity within the layer II under steady status. As the electroosmotic force is concentrated in the EDL region close to the wall, the change of velocity profiles mainly focuses in the EDL narrow region. In addition, with the increase in time, the EOF velocity approaches gradually steady status. That is to say, further increase in the time will lead to invariable velocity profile.

The influences of dielectric constant ratio $\varepsilon(0.3,0.5,0.7,1.1)$ of layer II to layer I on the transient EOF velocity of Maxwell fluids are illustrated in Fig. 6 for different times. When given the value of $D e_{2}$, the $D e_{1}$ increases due to $1 / D e_{1}=\varepsilon^{1 / 2} / D e_{2}$ and $\varepsilon$ adds, while high value of $D e_{1}$ results in large velocity. It 
(a) 0.8

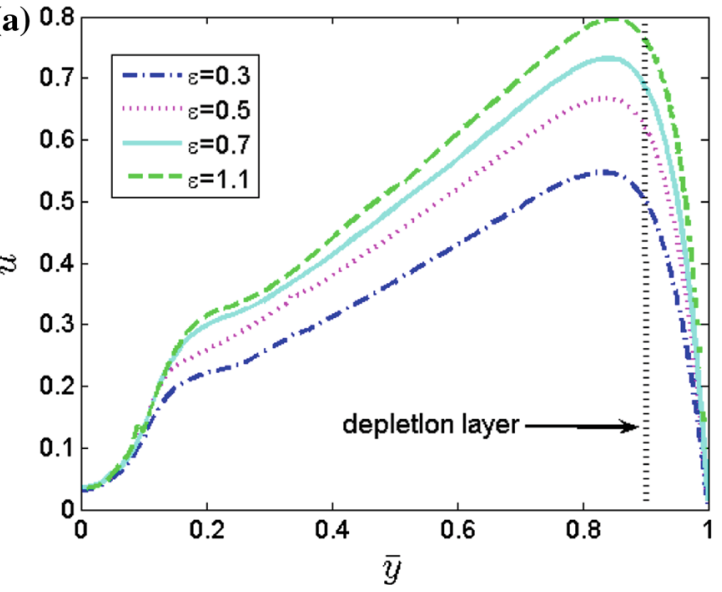

(c)

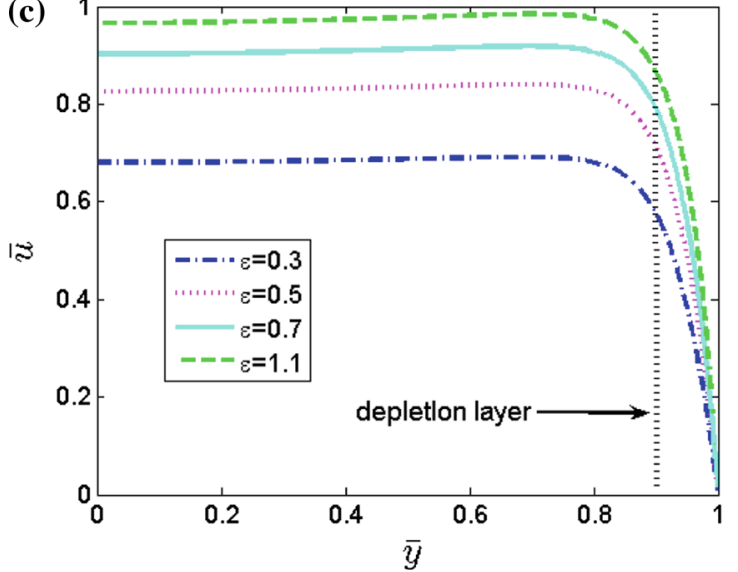

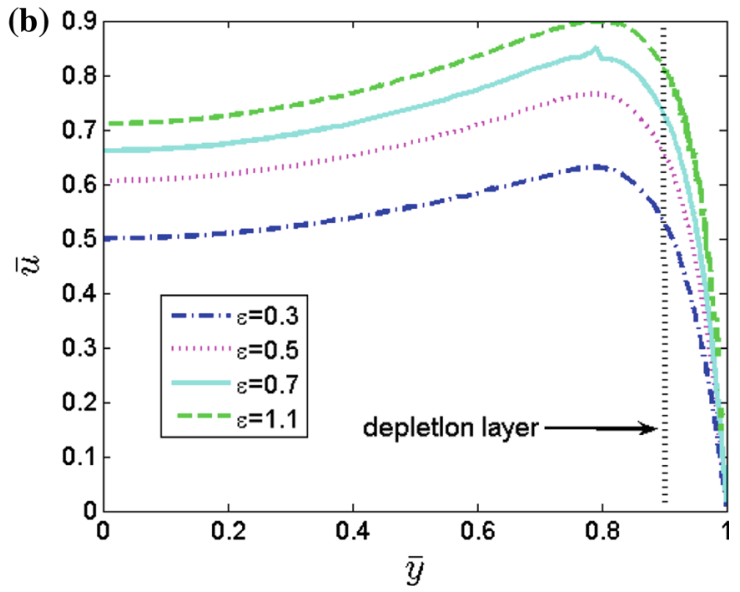

(d)

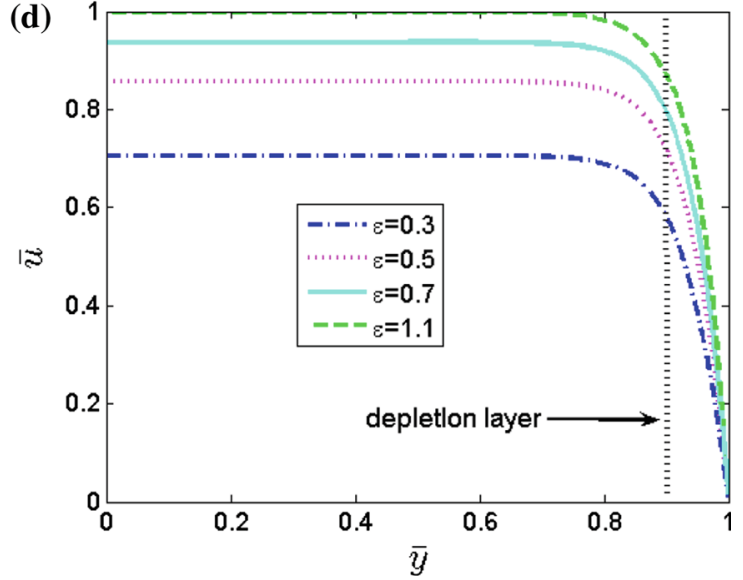

FIG. 6. Influences of dielectric constant ratio $\varepsilon$ on the transient EOF velocity of Maxwell fluids $\left(1 / D e_{2}=20,1 / D e_{1}=\right.$ $\left.\varepsilon^{1 / 2} / D e_{2}, \Delta \bar{\psi}=0, \rho=1, \bar{\lambda}_{1}=0.1, Q=0, \mu=1\right): \mathbf{a} t=0.3, \mathbf{b} t=0.6, \mathbf{c} t=1.2, \mathbf{d} t=2.0$

can be found that larger dielectric constant ratio leads to larger velocity under steady status. Similarly, with the increase in time, the EOF velocity approaches gradually steady status.

\section{Conclusion}

In this work, the transient EOF of generalized Maxwell fluids through a slit microchannel was investigated taking the depletion effects and Maxwell electrical stress at the interface into account. The overall flow is divided into depletion layer and bulk flow outside of depletion layer. Through solving the linearized Poisson-Boltzmann equation, the Cauchy momentum equation, and the Maxwell constitutive equation, semi-analytical solutions of these two layers were obtained by Laplace transform respectively. By numerical computations of inverse Laplace transform, the influences of viscosity ratio $\mu$, density ratio $\rho$, dielectric constant ratio $\varepsilon$ of layer II to layer I, relaxation time $\bar{\lambda}_{1}$, interface charge density jump $Q$, and interface zeta potential difference $\Delta \bar{\psi}$ on transient velocity amplitude are studied. The velocity behaves strong jump at the interface between layer I and layer II with the increase in interface charge density jump $Q$. The velocity amplitude becomes large with the interface zeta potential difference $\Delta \bar{\psi}$ under 
steady status. However, the velocity amplitude becomes small with the increase in the viscosity within the Maxwell fluid layer II under steady status. Larger dielectric constant ratio $\varepsilon$ leads to larger velocity under steady status. Finally, it is interesting to find that under steady status, the density ratio has little effect on the EOF velocity.

\section{Acknowledgments}

The work was supported by the National Natural Science Foundation of China (Grant Nos. 11062005, 11202092), Opening fund of State Key Laboratory of Nonlinear Mechanics, the Program for Young Talents of Science and Technology in Universities of Inner Mongolia Autonomous Region (Grant No. NJYT-13A02), the Natural Science Foundation of Inner Mongolia (Grant Nos. 2010BS0107, 2012MS0107), the research start-up fund for excellent talents at Inner Mongolia University (Grant No. Z20080211), the support of Natural Science Key Fund of Inner Mongolia (Grant No. 2009ZD01), the Innovative programs funded projects of Postgraduate Education in Inner Mongolia Autonomous Region, and Inner Mongolia University of enhancing the comprehensive strength funding (Grant No. 1402020201).

\section{References}

1. Stone, H.A., Stroock, A.D., Ajdari, A.: Engineering flows in small devices: microfluidics toward a Lab-on-a-chip. Ann. Rev. Fluid Mech. 36, 381-411 (2004)

2. Bayraktar, T., Pidugu, S.B.: Characterization of liquid flows in microfluidic systems. Int. J. Heat Mass Trans. 49, 815824 (2006)

3. Li, D.: Electrokinetics in Microfluidics. Elsevier, Amsterdam (2004)

4. Karniadakis, G., Beskok, A., Aluru, N.: Micorflows and Nanoflows: Fundamentals and Simulation. Springer, New York (2005)

5. Levine, S., Marriott, J.R., Neale, G., Epstein, N.: Theory of electrokinetic flow in fine cylindrical capillaries at high zeta potentials. J. Colloid Interface Sci. 52, 136-149 (1975)

6. Tsao, H.K.: Electroosmotic flow through an annulus. J. Colloid Interface Sci. 225, 247-250 (2000)

7. Hsu, J.P., Kao, C.Y., Tseng, S.J., Chen, C.J.: Electrokinetic flow through an elliptical microchannel: effects of aspect ratio and electrical boundary conditions. J. Colloid Interface Sci. 248, 176-184 (2002)

8. Yang, C., Li, D., Masliyah, J.H.: Modeling forced liquid convection in rectangular microchannels with electrokinetic effects. Int. J. Heat Mass Transf. 41, 4229-4249 (1998)

9. Bianchi, F., Ferrigno, R., Girault, H.H.: Finite element simulation of an electroosmotic driven flow division at a t-junction of microscale dimensions. Anal. Chem. 72, 1987-1993 (2000)

10. Wang, C.Y., Liu, Y.H., Chang, C.C.: Analytical solution of electro-osmotic flow in a semicircular microchannel. Phys. Fluids 20, 063105 (2008)

11. Dutta, P., Beskok, A.: Analytical solution of time periodic electroosmotic flows: analogies to Stokes' second problem. Anal. Chem. 73, 5097-5102 (2001)

12. Keh, H.J., Tseng, H.C.: Transient electrokinetic flow in fine capillaries. J. Colloid Interface Sci. 242, 450-459 (2001)

13. Kang, Y.J., Yang, C., Huang, X.Y.: Dynamic aspects of electroosmotic flow in a cylindrical microcapillary. Int. J. Eng. Sci. 40, 2203-2221 (2002)

14. Wang, X.M., Chen, B., Wu, J.K.: a semianalytical solution of periodical electro-osmosis in a rectangular microchannel. Phys. Fluids 19, 127101 (2007)

15. Chakraborty, S., Ray, S.: mass flow-rate control through time periodic electro-osmotic flows in circular microchannels. Phys. Fluids 20, 083602 (2008)

16. Jian, Y.J., Yang, L.G., Liu, Q.S.: Time periodic electro-osmotic flow through a microannulus. Phys. Fluids 22, $042001(2010)$

17. Deng, S.Y., Jian, Y.J., Bi, Y.H., Chang, L., Wang, H.J., Liu Q., S.: Unsteady electroosmotic flow of power-law fluid in a rectangular microchannel. Mech. Res. Commun. 39, 9-14 (2010)

18. Das, S., Chakraborty, S.: Analytical solutions for velocity, temperature and concentration distribution in electroosmotic microchannel flows in a non-Newtonian bio-fluid. Anal. Chim. Acta 559, 15-24 (2006)

19. Chakraborty, S.: Electroosmotically driven capillary transport of typical non-Newtonian biofluids in rectangular microchannels. Anal. Chim. Acta 605, 175-184 (2007) 
20. Zhao, C., Zholkovskij, E., Masliyah, J.H., Yang, C.: Analysis of electroosmotic flow of power-law fluids in a slit microchannel. J. Colloid Interface Sci. 326, 503-510 (2008)

21. Zhao, C., Yang, C.: Nonlinear Smoluchowski velocity for electroosmosis of power-law fluids over a surface with arbitrary zeta potentials. Electrophoresis 31, 973-979 (2010)

22. Tang, G.H., Li, X.F., He, Y.L., Tao, W.Q.: Electroosmotic flow of non-newtonian fluid in microchannels. J. Non-Newton. Fluid Mech. 157, 133-137 (2009)

23. Park, H.M., Lee, W.M.: Helmholtz-Smoluchowski velocity for viscoelastic electroosmotic flows. J. Colloid Interface Sci. 317, 631-636 (2008)

24. Park, H.M., Lee, W.M.: Effect of viscoelasticity on the flow pattern and the volumetric flow rate in electroosmotic flows through a microchannel. Lab Chip 8, 1163-1170 (2008)

25. Zhao, C., Yang, C.: Exact solutions for electro-osmotic flow of viscoelastic fluids in rectangular micro-channels. Appl. Math. Comput. 211, 502-509 (2009)

26. Afonso, A.M., Alves, M.A., Pinho, F.T.: Analytical solution of mixed electro-osmotic pressure driven flows of viscoelastic fluids in microchannels. J. Non-Newton. Fluid Mech. 159, 50-63 (2009)

27. Liu, Q.S., Jian, Y.J., Yang, L.G.: Time periodic electroosmotic flow of the generalized Maxwell fluids between two micro-parallel plates. J. Non-Newton. Fluid Mech. 166, 478-486 (2011)

28. Jian, Y.J., Liu, Q.S., Yang, L.G.: AC electroosmotic flow of generalized Maxwell fluids in a rectangular microchannel. J. Non-Newton. Fluid Mech. 166, 1304-1314 (2011)

29. Berli, C.L.A., Olivares, M.L.: Electrokinetic flow of non-Newtonian fluids on microchannels. J. Colloid Interface Sci. 320, 582-589 (2008)

30. Sousa, J.J., Afonso, A.M., Pinho, F.T.: Effect of the skimming layer on electro- osmotic-Poiseuille flows of viscoelastic fluids. Microfluid Nanofluid 10, 107-122 (2011)

31. Liu, Q.S., Jian, Y.J., Yang, L.G.: Alternating current electroosmotic flow of the Jeffreys fluids through a slit microchannel. Phys. Fluids 23, 102001 (2011)

32. Volkov, A.G., Deamer, D.W., Tanelian, D.L., Markin, V.S.: Electrical double layers at the oil/water interface. Prog. Surf. Sci. 53, 1-134 (1996)

33. Choi, W., Sharma, A., Qian, S.Z., Lim, G., Joo, S.W.: On steady two-fluid electroosmotic flow with full interfacial electrostatics. J. Colloid Interface Sci. 357, 521-526 (2008)

34. Mayur, M., Amiroudine, S., Lasseux, D.: Free-surface instability in electro-osmotic flows of ultrathin liquid films. Phys. Rev. E 85, 046301 (2012)

35. De Hoog, F.R., Knight, J.H., Stokes, A.N.: An improved method for numerical inversion of Laplace transforms. SIAM J. Sci. Stat. Comput. 3, 357-366 (1982)

Yongjun Jian, Jie Su, Long Chang and Quansheng Liu

School of Mathematical Science

Inner Mongolia University

Hohhot 010021

China

e-mail: jianyongjun@yahoo.com.cn

Long Chang

School of Mathematics and Statistics

Inner Mongolia University of Finance and Economics

Hohhot 010051

China

e-mail: suolunga@163.com

Guowei He

The State Key Laboratory of Nonlinear Mechanics (LNM)

Institute of Mechanics

Chinese Academy of Sciences

Beijing 100080

China

e-mail: hgw@lnm.imech.ac.cn

(Received: February 7, 2013; revised: May 13, 2013) 